\title{
Comparação entre diferentes processos oxidativos avançados para degradação de corante azo
}

\section{Comparison of various advanced oxidation processes for azo dye degradation}

\author{
Camila Costa de Amorim \\ Engenheira Ambiental. Doutoranda do Programa de Pós-Graduação em Saneamento, Meio Ambiente e Recursos Hídricos da Universidade Federal de Minas \\ Gerais (UFMG). Professora Assistente do Instituto de Ciências Exatas e Aplicadas da Universidade Federal de Ouro Preto (UFOP) \\ Mônica Maria Diniz Leão \\ Engenheira Química. Doutora em Engenharia Ambiental. Professora Associada do Departamento de Engenharia Sanitária e Ambiental da UFMG \\ Regina de Fátima Peralta Muniz Moreira \\ Engenheira Química. Doutora em Química. Professora Associada do Departamento de Engenharia Química e Engenharia de Alimentos da Universidade \\ Federal de Santa Catarina (UFSC)
}

\section{Resumo}

$\mathrm{O}$ objetivo deste estudo foi comparar os processos de oxidação avançada $\left(\mathrm{H}_{2} \mathrm{O}_{2}, \mathrm{UV}, \mathrm{UV} / \mathrm{H}_{2} \mathrm{O}_{2}, \mathrm{Fe}^{2+} / \mathrm{H}_{2} \mathrm{O}_{2}, \mathrm{UV} / \mathrm{Fe}^{2+} / \mathrm{H}_{2} \mathrm{O}_{2}\right)$ para descoloração do corante têxtil Reactive Red 195. Também foi investigada a utilização do pó do desempoeiramento da ala de corrida do alto forno como fonte alternativa de ferro na peroxidação catalítica, com e sem radiação UV, para degradação do corante. O efeito das concentrações de $\mathrm{H}_{2} \mathrm{O}_{2}$ e corante na cinética de descoloração foram estudados. Os resultados indicaram que a maior eficiência de descoloração foi obtida através do sistema foto-Fenton com o uso do resíduo; entretanto, a utilização do resíduo no sistema Fenton sem irradiação atingiu a mesma eficiência, com tempo de reação aumentado em apenas 15 minutos. A utilização do resíduo siderúrgico aumentou a velocidade de descoloração, mostrando-se bastante promissora como fonte de ferro.

Palavras-chave: $\mathrm{UV} / \mathrm{H}_{2} \mathrm{O}_{2}$, Fenton, foto-Fenton, reutilização de resíduo siderúrgico, Reactive Red 195, cinética de oxidação.

\section{Abstract}

This study aimed at comparing various advanced oxidation processes $\left(\mathrm{H}_{2} \mathrm{O}_{2}, \mathrm{UV}, \mathrm{UV} / \mathrm{H}_{2} \mathrm{O}_{2}, \mathrm{Fe}^{2+} / \mathrm{H}_{2} \mathrm{O}_{2}, \mathrm{UV} / \mathrm{Fe}^{2+} / \mathrm{H}_{2} \mathrm{O}_{2}\right)$ for textile dye Reactive Red 195 decolorization. The use of blast furnace dust (BFD) was also investigated as an alternative source of iron in catalytic peroxidation with and without UV radiation. The effects of $\mathrm{H}_{2} \mathrm{O}_{2}$ and dye concentrations in kinetics of decolorization were studied. Decolorization reactions follow pseudo-first order kinetics. The results indicated greater efficiency of decolorization in the photo-Fenton system with the use of BFD; however, the use of BFD in the Fenton system without irradiation reached the same efficiency with a reaction time only 15 minutes superior. The use of blast furnace dust increased considerably the rates of reactions and is very promising as a source of iron.

Keywords: $\mathrm{UV} / \mathrm{H}_{2} \mathrm{O}_{2}$, Fenton, photo-Fenton, reuse of blast furnace waste, Reactive Red 195, oxidation kinetics.

\section{Introdução}

As indústrias têxteis consomem grandes volumes de água e produtos químicos em seus processos de transformação. Os reagentes químicos utilizados são bastante diversificados na composição química, variando dos compostos inorgânicos aos polímeros e produtos orgânicos. Os efluentes líquidos das indústrias têxteis tipicamente são coloridos, devido ao uso extensivo dos corantes nos processos de tingimento e estamparia. (NIGAM et al, 2000). No tratamento dos efluentes líquidos, a degradação dos corantes é dificultada pela complexidade da estrutura molecular e sua origem sintética. Mais de 90\% dos 4.000 corantes testados pela Ecological and Toxicological Association of the Dyestuffs Manufacturing Industry (ETAD) apresentaram altos valores de toxicidade. As maiores taxas de toxicidade foram encontradas entre corantes diazo, básicos e diretos (ROBINSON et al, 2001) 
Corantes azoicos compreendem uma importante classe de compostos orgânicos sintéticos, que são caracterizados pela presença de uma ou mais ligações do tipo azo (-N=N-). Representam cerca de $50 \%$ da produção mundial de corantes e são amplamente utilizados em diferentes tipos de indústrias, tais como curtumes, indústrias têxteis, alimentícias, de cosméticos e de papel, sendo a indústria têxtil o maior consumidor (SLEIMAN et al, 2007). Além disso, alguns corantes azoicos e seus subprodutos, como as aminas aromáticas, são altamente cancerígenos (CICEK et al, 2007). As aminas aromáticas são formadas como metabólitos da clivagem redutiva das ligações azo, e muitas vezes são mais tóxicas do que as moléculas intactas dos corantes (AZBAR et al, 2007).

O lançamento desses efluentes em ambientes naturais é bastante problemático tanto para a vida aquática quanto para os humanos. O tratamento biológico não apresenta uma solução completa para o problema devido à baixa biodegradabilidade de muitos corantes (LUCAS; PERES, 2006). Em geral, as indústrias têxteis no Brasil tratam seus efluentes empregando a tecnologia de lodos ativados, sendo necessária a aplicação de tratamentos adicionais para a redução da cor. Outras formas de eliminação dos corantes se baseiam na utilização de processos integrados que envolvem combinações entre processos biológicos, físicos e químicos (GALINDO et al, 2001; ROBINSON et al, 2001).

Técnicas como a adsorção (CICEK et al, 2007), adsorção em carvão ativado (ÓRFÃO et al, 2006), coagulação/floculação (LEE et al, 2006; PAPIC et al, 2004), ultrafiltração (ZAGHBANI et al, 2007) e osmose reversa (ŠOSTAR-TURK et al, 2005) têm sido relatadas para a remoção de corantes têxteis. No entanto, além de serem mais dispendiosas do que os processos biológicos, realizam apenas a transferência de fase do poluente, e necessitam de pós-tratamento dos resíduos sólidos gerados ou regeneração do material adsorvente.

Os processos oxidativos avançados (POA) têm sido descritos como alternativa para a remoção de poluentes persistentes e de efluentes com elevada carga orgânica, quando os tratamentos convencionais não alcançam a eficiência necessária. Os POA se baseiam em processos físico-químicos capazes de produzir alterações profundas na estrutura química dos poluentes, e são definidos como processos envolvendo a geração e uso de agentes oxidantes fortes, principalmente radicais hidroxila (HO*) (TARR, 2003). Em águas residuárias coloridas, os radicais hidroxila atacam as ligações azo insaturadas do cromóforo, descolorindo assim o efluente (HSUEH et al, 2005).

Devido ao elevado poder oxidante dos radicais hidroxila, inferior apenas ao flúor, os POA têm sido utilizados com um interesse crescente. A característica comum de todos os POA é o uso dos radicais livres reativos, principalmente os radicais hidroxila, que podem ser gerados por vários métodos, incluindo processos com $\mathrm{H}_{2} \mathrm{O}_{2}$ ou ozônio, ou reações induzidas pela luz (TARR, 2003; OLIVER et al, 2000). Esses radicais atacam moléculas orgânicas pela abstração de um átomo de hidrogênio ou pela adição às duplas ligações. $\mathrm{O}$ mecanismo mais aceito para a degradação de um composto orgânico genérico (R) pelo radical hidroxila pode ser representado de acordo com as Equações 1 a 4 .

$\mathrm{HO}^{\bullet}+\mathrm{RH} \rightarrow \mathrm{H}_{2} \mathrm{O}+\mathrm{R}^{\bullet}$

$\mathrm{R}^{\cdot}+\mathrm{H}_{2} \mathrm{O}_{2} \rightarrow \mathrm{ROH}+\mathrm{HO}^{\bullet}$

$\mathrm{R}^{\cdot}+\mathrm{O}_{2} \rightarrow \mathrm{ROO}^{\bullet}$

$\mathrm{ROO}{ }^{\bullet}+\mathrm{RH} \rightarrow \mathrm{ROOH}+\mathrm{R}^{\bullet}$

Esses radicais podem ser produzidos utilizando-se agentes oxidantes como o ozônio, o peróxido de hidrogênio, além da radiação $\mathrm{UV}$, ou de combinações como $\mathrm{O}_{3} / \mathrm{H}_{2} \mathrm{O}_{2}, \mathrm{O}_{3} / \mathrm{UV}, \mathrm{H}_{2} \mathrm{O}_{2} / \mathrm{UV}, \mathrm{O}_{3} / \mathrm{H}_{2} \mathrm{O}_{2} /$ UV, e da combinação de peróxido de hidrogênio com íons ferrosos no chamado Reagente de Fenton (AZBAR et al, 2004).

A geração catalítica dos radicais hidroxila utilizando $\mathrm{Fe}^{2+}$ é bem conhecida e esquematicamente representada pelas Equações 5 e 6 .

$\mathrm{Fe}^{2+}+\mathrm{H}_{2} \mathrm{O}_{2} \rightarrow \mathrm{Fe}^{3+}+\mathrm{HO}^{\cdot}+\mathrm{OH}$

$\mathrm{Fe}^{3+}+\mathrm{H}_{2} \mathrm{O}_{2} \rightarrow \mathrm{Fe}^{2+}+\mathrm{H}^{+}+\mathrm{HO}_{2}$

Embora os processos oxidativos apresentem significativas vantagens sobre os métodos convencionais de tratamento, um dos obstáculos para a aplicação dos POA em larga escala tem sido atribuído ao elevado custo dos reagentes, e ao custo operacional envolvendo as fontes de energia como a luz ultravioleta (GALINDO et al, 2001). A utilização de óxidos de ferro como catalisadores no processo Fenton é denominado de Fenton heterogêneo, e diversas pesquisas têm sido relatadas nessa área, inclusive para remoção de corantes (ANDREOZZI et al, 2002; CHOU et al, 2001; CENTI et al, 2000; DANTAS et al, 2006). Resíduos sólidos com alto teor de ferro podem ser boas fontes de catalisadores em sistemas heterogêneos, diminuindo o consumo de sais de ferro, além da possibilidade de seu reuso.

O pó do desempoeiramento da ala de corrida do alto forno é um resíduo da indústria siderúrgica, e seus principais constituintes são os óxidos de ferro, carvão ou coque, fundentes e refratários (silício, cálcio, magnésio e óxidos elementares em menor quantidade). A reciclagem direta desse resíduo geralmente não é possível, uma vez que contém alguns elementos indesejáveis, como zinco, chumbo e metais alcalinos, que podem causar dificuldades operacionais nos altos fornos. A eliminação desses resíduos exige procedimentos especiais, devido às suas características químicas, especialmente com relação ao teor de metais, sendo a reutilização e reciclagem desses resíduos opções mais atraentes.

O principal objetivo deste trabalho foi avaliar a aplicabilidade de diferentes tecnologias avançadas de oxidação $\left(\mathrm{H}_{2} \mathrm{O}_{2}, \mathrm{UV}, \mathrm{H}_{2} \mathrm{O}_{2} / \mathrm{UV}\right.$, $\left.\mathrm{Fe}^{2+} / \mathrm{H}_{2} \mathrm{O}_{2}, \mathrm{Fe}^{2+} / \mathrm{H}_{2} \mathrm{O}_{2} / \mathrm{UV}\right)$ na descoloração do corante Reactive Red 195 (RR195), do tipo azo e extensivamente utilizado nas indústrias têxteis brasileiras, e testar a eficácia do uso do pó do desempoeiramento da ala de corrida do alto forno como catalisador na reação de Fenton e foto-Fenton heterogêneos. 


\section{Materiais e métodos}

A solução aquosa de corante RR195, cuja estrutura molecular é mostrada na Figura 1, foi preparada pela dissolução do corante em água destilada. Esse corante foi selecionado neste trabalho pelo fato de ser extensivamente utilizado na indústria têxtil e pelo fato de já existirem mecanismos propostos para sua degradação (JIRASRIPONGPUN et al, 2007; SILVA et al, 2006).

$\mathrm{O}$ pH da solução aquosa era ajustado ao valor desejado, utilizando $\mathrm{NaOH}$, ou $\mathrm{H}_{2} \mathrm{SO}_{4}$, ambos $1 \mathrm{~N}$.

Foi utilizado peróxido de hidrogênio p.a. 30\% (m/v), marca Merck. Todos os demais reagentes utilizados eram de pureza analítica.

O catalisador sólido utilizado nas reações heterogêneas foi o pó do desempoeiramento da ala de corrida do alto forno (PAF), utilizado sem qualquer tratamento prévio. Sua caracterização foi realizada pela determinação da área superficial BET (DABROWSKI, 2001) e da distribuição do tamanho dos poros e volume (Quantachrome Autosorb Automated Gas Sorption, NOVA1200, versão 5.25). Os minerais e elementos presentes no resíduo foram identificados por difração e fluorescência de raios X (modelo X'PERT, marca Philips). A morfologia foi analisada por microscopia eletrônica de varredura (modelo XL30, marca Phillips). Para obtenção do ponto de carga zero, utilizou-se o método citado por Rivera-Utrilla (2000).

A cinética das reações na ausência de luz foram realizadas em béqueres de $1 \mathrm{~L}$, protegidos da luz, num equipamento de jar test (Q305M, Quimis) com velocidade de agitação de $120 \mathrm{rpm}$, em pH = 3,0. À solução aquosa do corante de concentração inicial conhecida, era adicionado peróxido de hidrogênio, em concentração na faixa de 20 a 100 mg. $L^{-1}$. A concentração de Fe total na solução variou no intervalo de 0 a $15 \mathrm{mg} \cdot \mathrm{L}^{-1}$. Em intervalos regulares de tempo, uma alíquota da solução era retirada e filtrada, e a determinação da concentração do corante remanescente era realizada espectrofotometricamente (espectrofotômetro UV-1650 PC, Shimadzu), no comprimento de onda de maior absorbância do corante $\left(\lambda_{\text {máx }}=517 \mathrm{~nm}\right)$, através de uma curva de calibração (absorvância versus concentração do corante) previamente determinada.

As reações realizadas sob irradiação UV foram realizadas num reator fotoquímico anular, descrito em detalhes por Sauer et al (2002). A irradiação UV era fornecida por uma lâmpada de médio vapor de mercúrio de $80 \mathrm{~W}$, localizada no centro do reator cilíndrico. O reator era encamisado e a temperatura da reação era mantida em $25^{\circ} \mathrm{C}$. Para a determinação do fluxo de radiação da lâmpada, foi utilizado o método de actinometria de ferrioxalato de potássio de acordo com Murov (1973), citado por Sauer et al (2002).

A dosagem de catalisador heterogêneo em todos os testes era 1 g. $\mathrm{L}^{-1}$. O sólido era adicionado à solução aquosa do corante, o pH ajustado ao valor desejado $(3,0)$ e a suspensão era mantida sob agitação constante por tempo suficientemente longo (30 horas) para que se atingisse o equilíbrio da adsorção. Testes prévios indicaram que o equilíbrio de adsorção era atingido em menos de 30 horas. Em seguida, era adicionado o peróxido de hidrogênio na concentração desejada (20 e 100 mg. $\mathrm{L}^{-1}$ ) e iniciada a reação. Esse procedimento garantia que toda descoloração medida era devida unicamente às reações de degradação do corante. Em intervalos regulares de tempo, uma alíquota da solução era retirada, filtrada em membrana PVDF com diâmetro do poro 0,22 ㅆm (GVWP01300, Milipore), sendo em seguida medida a concentração remanescente do corante.

As análises de determinação da concentração de ferro total e solúvel foram realizadas pelo método colorimétrico com ortofenantrolina (APHA, 2005). Para eliminar a interferência da cor, essas análises foram realizadas depois de terminada a reação de descoloração no processo Fenton.

\section{Resultados}

\section{Caracterização do resíduo}

A área superficial para o resíduo siderúrgico em estudo é relativamente pequena quando comparada com os valores obtidos para sólidos comumente utilizados como adsorventes (Tabela 1). Órfão et al (2006) relatam uma área superficial BET da ordem de $1.100 \mathrm{~m}^{2} \cdot \mathrm{g}^{-1}$ para o carvão ativado utilizado em sua pesquisa de adsorção de corantes reativos. Wang e Zhu (2006) utilizaram zeólitas naturais para remoção de corantes têxteis e encontraram como características desse sólido uma área superficial BET de $16 \mathrm{~m}^{2} \cdot \mathrm{g}^{-1}$, volume do poro de 3,9 x $10^{-2} \mathrm{~cm}^{3} \cdot \mathrm{g}^{-1}$ e tamanho da partícula $<75 \mu \mathrm{m}$.

De fato, testes prévios mostraram que a capacidade de adsorção do corante sobre o PAF é desprezível, na faixa de $\mathrm{pH}$ de 3 a 7 .

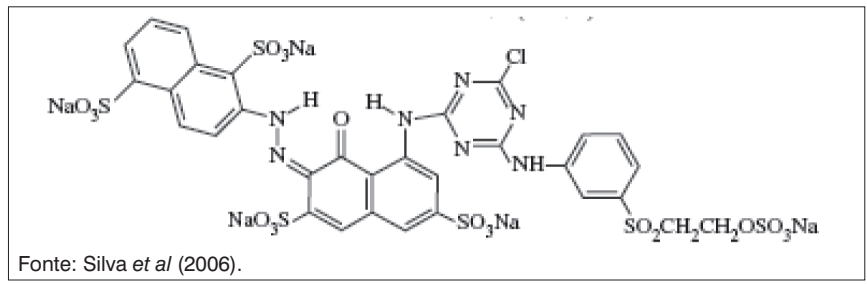

Figura 1 - Fórmula estrutural do corante RR195.

Tabela 1 - Resultados da caracterização do resíduo siderúrgico

\begin{tabular}{lcc} 
Parâmetros & Valores \\
\hline Área BET $\left(\mathrm{m}^{2} \cdot \mathrm{g}^{-1}\right)$ & 3,4 \\
\hline Volume total do poro $\left(\mathrm{cm}^{3} \cdot \mathrm{g}^{-1}\right)$ & $1,11 \times 10^{-3}$ \\
\hline Diâmetro do poro $(\mathrm{nm})$ & 13,2 \\
\hline Tamanho das partículas & & $99,6 \%<75 \mu \mathrm{m}$ \\
$\mathrm{pH}_{\text {pcz }}$ & 10 \\
\hline Fluorescência de raios X & $\begin{array}{l}\text { Elementos majoritários: } \\
\text { Elementos minoritários: }\end{array}$ & $\mathrm{Fi}, \mathrm{Al}, \mathrm{S}, \mathrm{P}, \mathrm{Mg}$ \\
& Elementos-traço: & $\mathrm{Mn}, \mathrm{Cr}, \mathrm{Ti}, \mathrm{V}, \mathrm{Ca}, \mathrm{K}$, \\
& & $\mathrm{Cl}, \mathrm{Na}$
\end{tabular}



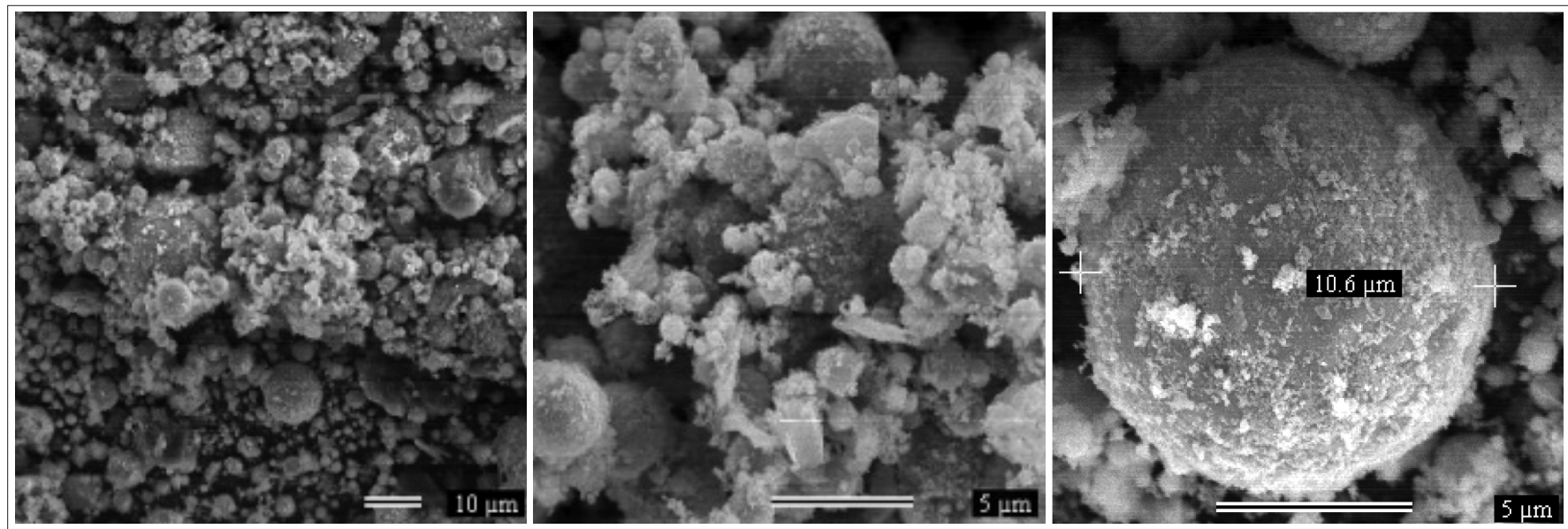

Figura 2 - Morfologia da superfície do PAF determinada por microscopia eletrônica de varredura (MEV).

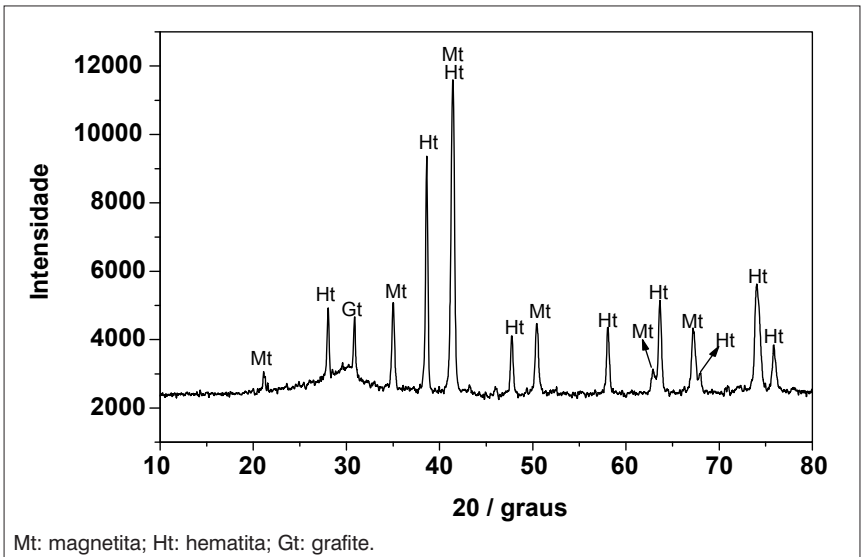

Figura 3 - Análise de difração de raios-X (DRX) do PAF.

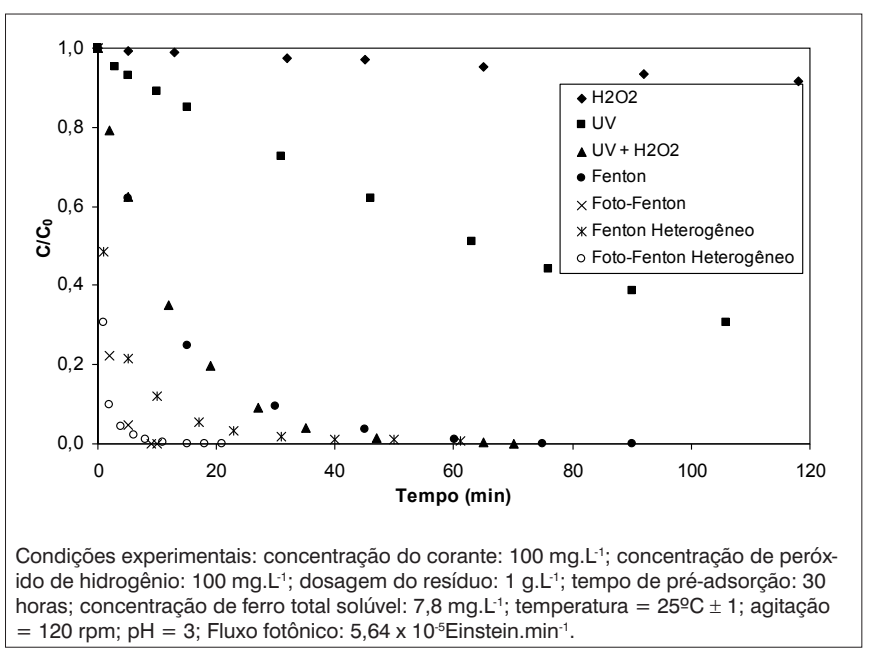

Figura 4 - Decomposição do Corante RR195 utilizando diferentes POA.

O ponto de carga zero $\left(\mathrm{pH}_{\mathrm{pcz}}\right)$ do PAF (Tabela 1) sugere que a adsorção de compostos aniônicos, como o corante RR195, seria favorecida para valores de $\mathrm{pH}$ menores que 10. Entretanto, estudos anteriores revelaram que a capacidade de adsorção do corante é extremamente pequena, mesmo em pH <10 (AMORIM et al, 2007).
A morfologia das partículas do PAF é mostrada na Figura 2, em que se observam partículas aproximadamente esféricas.

Os resultados da difração de raios X (Figura 3) revelaram a presença dos óxidos de ferro nas formas cristalinas hematita $e$ magnetita no resíduo siderúrgico, favorecendo a sua aplicação como catalisador no processo Fenton, devido à existência das formas de $\mathrm{Fe}^{2+}$ e $\mathrm{Fe}^{3+}$ presentes nesses óxidos de ferro. Goetita $(\alpha-\mathrm{FeOOH})$, lepdocrocita $(\gamma-\mathrm{FeOOH})$, hematita $\left(\mathrm{Fe}_{3} \mathrm{O}_{4}\right)$ e magnetita $\left(\mathrm{Fe}_{2} \mathrm{O}_{3}\right)$ têm sido efetivamente utilizadas como catalisadores heterogêneos no processo Fenton (DANTAS et al, 2006; CHOU et al, 2001; ANDREOZZI et al, 2002). No difratograma de raio-X aparece também um sinal em $30,8^{\circ}$, relacionado à presença de carbono na forma de grafite.

\section{Descoloração do corante RR195 utilizando diferentes POA}

A cinética de descoloração do corante RR195 utilizando diferentes POA é mostrada na Figura 4. Embora o peróxido de hidrogênio na concentração de $100 \mathrm{mg} \cdot \mathrm{L}^{-1}$ provoque a descoloração da solução aquosa contendo o corante na concentração de $100 \mathrm{mg}$. $\mathrm{L}^{-1}$, esta é inferior a 10\% após duas horas de reação.

A radiação UV foi capaz de provocar a degradação do corante RR195, porém com menor velocidade quando comparado com os outros tratamentos em estudo, alcançando, ao final de 120 minutos, remoção de $75 \%$ nas condições experimentais empregadas.

A cinética da reação de degradação pela associação $\mathrm{UV} / \mathrm{H}_{2} \mathrm{O}_{2}$ é mais rápida do que as reações sob UV ou com $\mathrm{H}_{2} \mathrm{O}_{2}$, alcançando completa descoloração e remoção total da concentração do corante em aproximadamente 50 minutos, com velocidade de reação similar ao processo Fenton.

Comparando os processos Fenton heterogêneo e homogêneo, percebe-se que o uso do PAF como fonte de ferro aumentou a velocidade da reação, confirmando assim a sua eficiência como catalisador. 
Cumpre ressaltar que o contato do PAF com a solução aquosa do corante garante que a adsorção possa ser desconsiderada. Além disso, esse contato da solução aquosa com o PAF antes da adição do peróxido de hidrogênio permite também que ocorra a lixiviação de íons ferro para a fase líquida, o que torna possível a ocorrência das reações Fenton em fase líquida. O processo heterogêneo foi capaz de remover $80 \%$ da cor em apenas cinco minutos de reação, chegando à completa descoloração em 35 minutos.

Os tratamentos combinados com luz UV, foto-Fenton homogêneo e heterogêneo foram mais eficientes que os demais processos em análise, sendo que $80 \%$ da cor é removida em cerca de dois minutos de reação. Esse resultado era esperado, uma vez que as reações fotocatalíticas possuem velocidades de reação maiores que as outras reações em estudo.

A Figura 5 resume a eficiência de cada tratamento aplicado à descoloração do corante RR195 após duas horas de reação, e o tempo necessário para completa remoção da cor da solução aquosa. Observa-se que o uso do PAF, no processo chamado Fenton heterogêneo, resulta de modo semelhante aos processos foto-Fenton e foto-Fenton heterogêneo.

A determinação da concentração de íons ferro na mistura reacional revelou que houve lixiviação do ferro para a fase líquida. Sendo assim, as reações de Fenton foram comparadas entre amostras filtradas e com o catalisador sólido após lixiviação. A maior parte da reação ocorre devido à presença do ferro solúvel, uma vez que não houve diferença na remoção de cor entre as duas amostras analisadas (filtrado e com PAF). Entretanto, a velocidade de descoloração do corante é maior quando o PAF é utilizado como fonte de ferro, quando comparada com o processo Fenton clássico, de acordo com os resultados da cinética de descoloração. A análise da presença de metais por absorção atômica, na solução aquosa de corante após o tratamento com Fenton utilizando o resíduo siderúrgico (dados não apresentados), indicaram resultados menores que o limite de detecção para $\mathrm{Cr}, \mathrm{Al}, \mathrm{Pb}, \mathrm{Cd}$ e $\mathrm{Cu}$, além de quantidades menores que 0,5 mg. $\mathrm{L}^{-1}$ para $\mathrm{Mg}, \mathrm{Mn}, \mathrm{Ni}$ e Zn. Esses resultados excluem a possibilidade do aumento da velocidade de descoloração pela lixiviação de outros metais ativos para o Fenton, como Mn, $\mathrm{Cr}$ e $\mathrm{Cu}$, e reforça a possibilidade de uma pequena contribuição das reações heterogêneas na superfície do sólido, além do fato da lenta liberação dos íons metálicos contribuírem para esse aumento de velocidade, conforme relatado por outros autores (MECOZZI et al, 2006; CHOU et al, 2001; ANDREOZZI et al, 2002).

\section{Influência da concentração de peróxido de hidrogênio na cinética de descoloração}

O aumento da concentração de peróxido de hidrogênio, de 20 a $100 \mathrm{mg} . \mathrm{L}^{-1}$ aumentou a velocidade de degradação, como mostrado na Figura 6. De acordo com a Figura 6A, a reação de descoloração não é altamente dependente da concentração de peróxido de hidrogênio para o tratamento combinado $\mathrm{UV} / \mathrm{H}_{2} \mathrm{O}_{2}$. Para tempos de reação acima de 30 minutos, a velocidade de descoloração é praticamente igual para as concentrações de 40 a 100 mg. L-1 $^{-1}$ de $\mathrm{H}_{2} \mathrm{O}_{2}$, levando à conclusão de que pode-se aplicar o tratamento com concentrações de peróxido de hidrogênio de 40 mg. $\mathrm{L}^{-1}$, para a concentração de corante igual a $100 \mathrm{mg} \cdot \mathrm{L}^{-1}$, otimizando assim o custo do tratamento.

Para processos Fenton (Figura 6B) e Fenton heterogêneo (Figura 6C), a reação de descoloração se mostrou dependente da concentração de peróxido de hidrogênio. A velocidade da reação aumenta à medida que se aumenta a concentração de peróxido de hidrogênio, devido à maior produção dos radicais hidroxila. No Fenton heterogêneo (Figura 6C), para concentrações de $\mathrm{H}_{2} \mathrm{O}_{2}$ entre 40 e $80 \mathrm{mg} \cdot \mathrm{L}^{-1}$ a remoção de cor é praticamente a mesma; além disso, para tempos de reação maiores que 45 minutos, com exceção da menor concentração de peróxido de hidrogênio (20 mg. $\left.\mathrm{L}^{-1}\right)$, todas as outras concentrações de peróxido de hidrogênio alcançaram mais de 98\% de eficiência de descoloração. O mesmo foi observado no processo Fenton (Figura 6B), mas observou-se que a velocidade da reação é menor, comprovado através dos estudos cinéticos apresentados na Figura 7

As Figuras 6D e 6E mostram que, para os processos fotoFenton e foto-Fenton heterogêneo, assim como nos sistemas sem radiação UV, a velocidade de descoloração aumenta à medida que se aumenta a concentração de peróxido de hidrogênio. Para concentrações de $\mathrm{H}_{2} \mathrm{O}_{2}$ entre 60 e $100 \mathrm{mg} \cdot \mathrm{L}^{-1}$, no processo Foto Fenton heterogêneo (Figura 6D), a remoção de cor é praticamente a mesma (maior que $90 \%$ ), em cerca de dois minutos. Para tempos de reação maiores que dez minutos, com exceção da concentração de $20 \mathrm{mg} \cdot \mathrm{L}^{-1}$, todas as concentrações de peróxido de hidrogênio utilizadas resultam em mais de 95\% de descoloração. Esses resultados permitem concluir que podem ser aplicados tratamentos com concentrações de $\mathrm{H}_{2} \mathrm{O}_{2}$ da ordem de $60 \mathrm{mg}$. $\mathrm{L}^{-1} \mathrm{com}$ tempos de reação baixos para a concentração de corante igual a $100 \mathrm{mg} \cdot \mathrm{L}^{-1}$

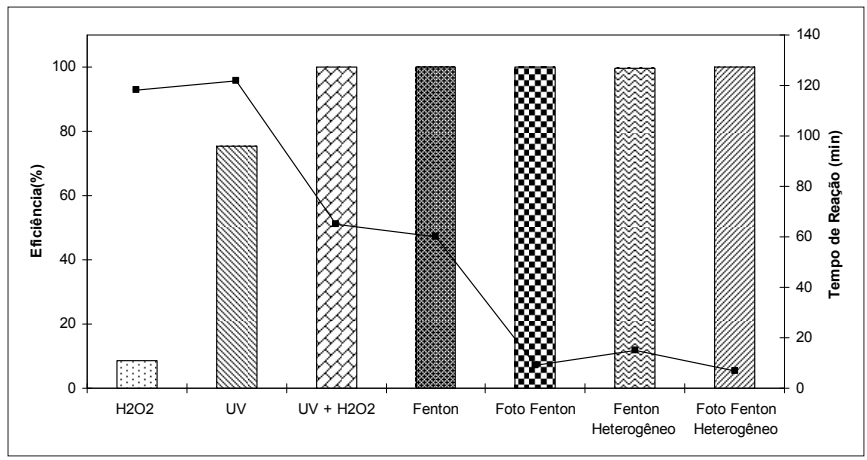

Figura 5 - Comparação entre as eficiências de descoloração dos diferentes processos oxidativos avançados estudados (barras), e os respectivos tempos de reação (linha contínua). 


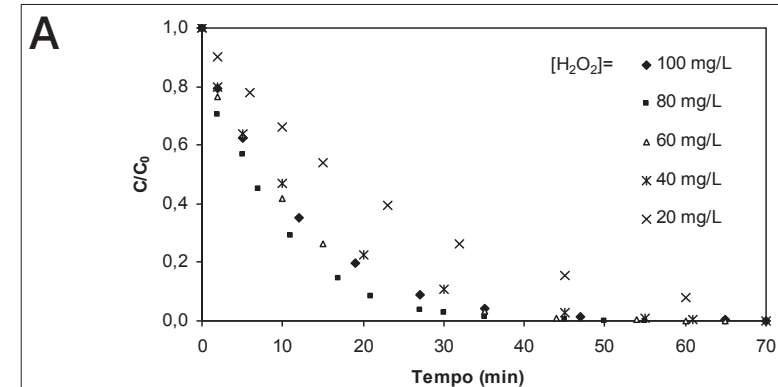

B

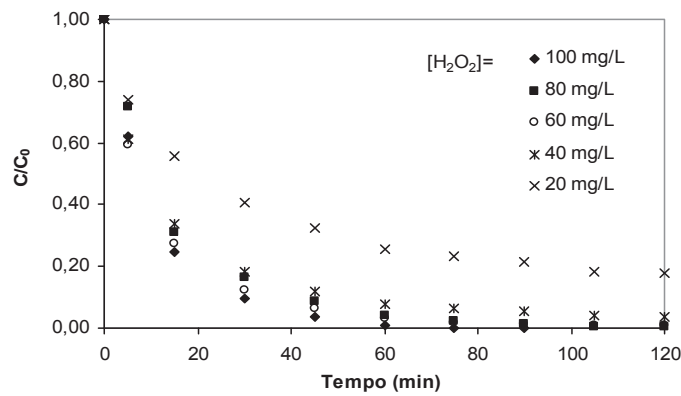

C

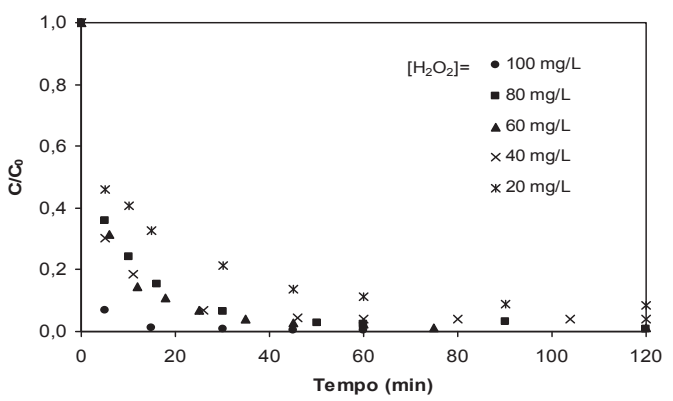

D

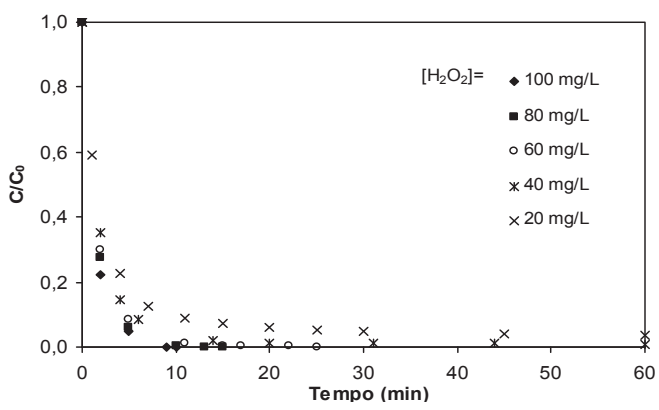

E

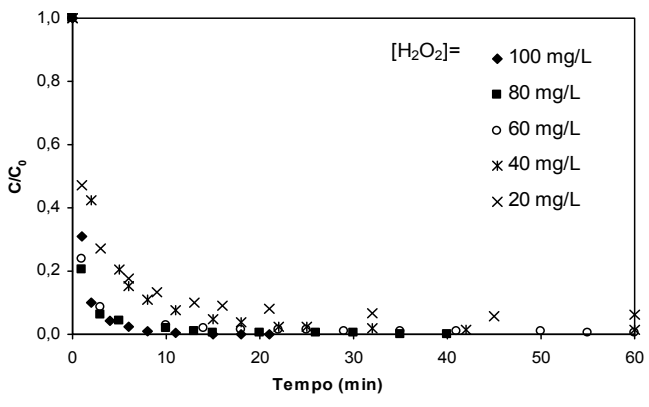

Condições experimentais: concentração do corante: $100 \mathrm{mg} \cdot \mathrm{L}^{-1}$; concentrações de peróxido de hidrogênio: 20 a $100 \mathrm{mg}^{-\mathrm{L}^{-1}}$; dosagem do resíduo $1 \mathrm{~g} / \mathrm{L}$; tempo de adsorção: 31 horas; concentração média de ferro total solúvel:13,0 mg. $\mathrm{L}^{-1}$; temperatura $=25^{\circ} \mathrm{C} \pm 1$; agitação = $120 \mathrm{rpm} ; \mathrm{pH}=3$.

Figura 6 - Efeito da concentração de peróxido de hidrogênio na decomposição do Corante RR195 através das reações de UV $+\mathrm{H}_{2} \mathrm{O}_{2}$ (A); Fenton (B); Fenton heterogêneo (C); foto-Fenton (D); foto-Fenton heterogêneo (E).

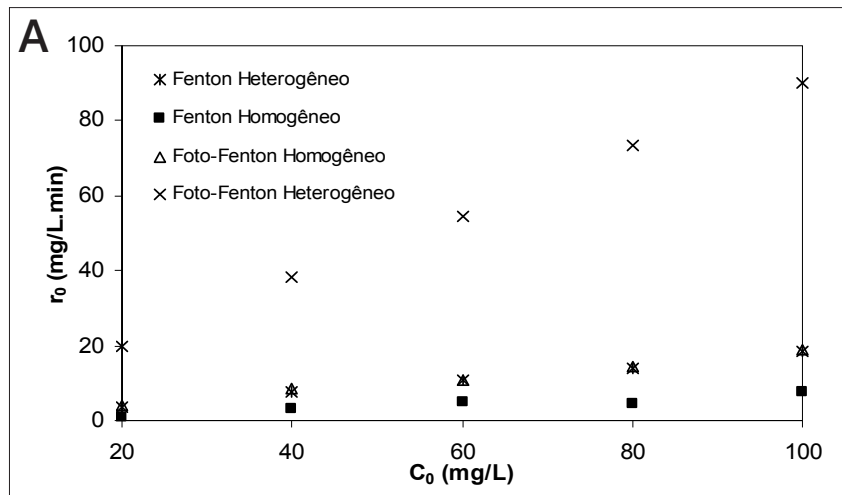

B

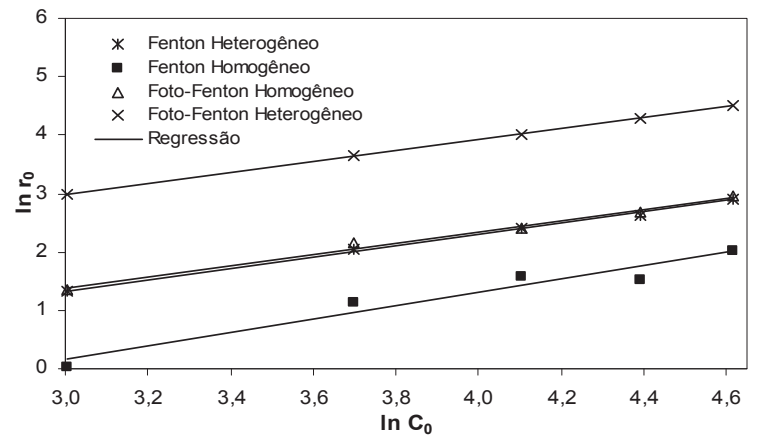

Condições experimentais: concentração do corante $=100 \mathrm{mg} \cdot \mathrm{L}^{-1}$; concentração de Ferro Total Solúvel. 13,9 mg. $\mathrm{L}^{-1}$; temperatura $=25^{\circ} \mathrm{C}+1 ; \mathrm{pH}=3,0$.

Figura 7 - (A) Velocidades iniciais das reações de descoloração do corante RR195 através dos tratamentos com Fenton e foto-Fenton; (B) Determinação da ordem das reações de descoloração através dos tratamentos com Fenton e foto-Fenton.

\section{Cinética de descoloração do RR195}

Nos processos Fenton (homogêneo e heterogêneo) com e sem irradiação, a concentração de peróxido de hidrogênio exerceu influência positiva sobre a descoloração do RR195.

Para determinar a ordem das reações e suas velocidades específicas (k), utilizou-se o método das velocidades iniciais: uma série de experimentos foi realizada com diferentes concentrações iniciais do corante $\left(C_{0}\right)\left(m g . L^{-1}\right)$, e a velocidade inicial de reação $\left(-r_{0}\right)\left(m g . L^{-1}\right.$. min $^{-1}$ ) foi determinada para cada ensaio. As outras variáveis, como dosagem de catalisador, concentração de ferro total solúvel, temperatura, pH e intensidade da radiação ultravioleta, foram mantidas constantes. A velocidade inicial $\left(-_{0}\right)$ foi calculada pela diferenciação numérica da curva de decaimento da concentração do corante em função do tempo, e extrapolando-se o resultado para o tempo zero. Assumindo que a lei de velocidade é da forma da Equação (7), o gráfico de $\ln \left(-r_{0}\right)$ em função de $\ln \left(C_{0}\right)$ será linear, com inclinação $(\alpha)$ igual à ordem da reação, e o intercepto igual ao valor de ln $\mathrm{k}$. $-\mathrm{r}_{0}=\mathrm{k} \cdot \mathrm{C}_{0}^{\alpha}$

A vantagem de se utilizar esse método reside na possibilidade de excluir possíveis interferências advindas das outras reações que ocorrem em paralelo com a reação em estudo, avaliando, dessa forma, a 
cinética da reação de acordo com apenas uma variável - no caso, a concentração inicial $C_{0}$.

A velocidade inicial da remoção de cor $\left(-r_{0}\right)$ em função da concentração inicial de peróxido de hidrogênio $\left(C_{0}\right)$ para os diferentes tratamentos com Fenton e foto-Fenton é mostrada na Figura 7A. É possível observar na Figura 7B a relação linear entre $\ln \left(-r_{0}\right)$ e $\ln \left(C_{0}\right)$, caracterizando uma cinética de pseudo-primeira ordem, cujos parâmetros são apresentados na Tabela 2.

Para todos os sistemas em estudo (Fenton, Fenton heterogêneo, fotoFenton e foto-Fenton heterogêneo), a cinética de descoloração do corante RR195 ocorre de acordo com o modelo de pseudo-primeira ordem. A maior velocidade específica de reação (k) foi encontrada para o processo foto-Fenton heterogêneo, sendo que os tratamentos foto-Fenton homogêneo e Fenton heterogêneo apresentam praticamente a mesma velocidade de reação. O tratamento com Fenton homogêneo obteve a menor taxa de remoção, comparado aos outros processos avaliados.

\section{Conclusões}

Entre os diferentes processos oxidativos avançados avaliados para a degradação do corante RR195, os que apresentaram melhores resultados foram os processos Fenton e foto-Fenton heterogêneos, utilizando o resíduo siderúrgico como catalisador. O processo UV/ $\mathrm{H}_{2} \mathrm{O}_{2}$ também se mostrou adequado para remoção do RR195, alcançando alta eficiência de remoção de cor em um período de tempo relativamente baixo. Quanto à natureza das reações que ocorrem quando o PAF foi usado
Tabela 2 - Constantes experimentais obtidas pela descoloração do RR195

\begin{tabular}{lccc} 
Tratamento & $\mathrm{n}^{*}$ & $\mathrm{k}^{* *}\left(\mathrm{~min}^{-1}\right)$ & $\mathrm{r}^{2}$ \\
\hline Fenton homogêneo & 1,150 & 0,037 & 0,942 \\
Fenton heterogêneo & 0,965 & 0,211 & 0,997 \\
Foto-Fenton homogêneo & 0,958 & 0,227 & 0,991 \\
Foto-Fenton heterogêneo & 0,943 & 1,171 & 0,100
\end{tabular}

como catalisador, observou-se que a maior parte da reação ocorre na fase líquida, com os íons ferro que são lixiviados do resíduo, agindo como catalisadores na reação Fenton. No entanto, o uso do PAF conduz à cinética de reação mais rápida, desde que haja pré-contato do sólido com a solução aquosa, e indica que reações heterogêneas podem também ocorrer na fase sólida, uma vez que o processo Fenton resulta em reações mais lentas de degradação do corante. A utilização prática do resíduo siderúrgico mostrou-se bastante promissora, uma vez que não representa perigo ambiental no que diz respeito à lixiviação de outros metais presentes, aumenta as taxas de reação e permite fácil separação sólido-líquido, além de eliminar custos no tratamento, substituindo a fonte de sais de ferro do sistema Fenton tradicional.

\section{Agradecimentos}

Os autores agradecem à Capes pela concessão das bolsas e apoio financeiro do Procad 0064050, à Fapemig (processo TEC1123/06) pelo apoio financeiro, ao CNPq pela concessão da bolsa de doutorado, e à Gerdau Açominas por ceder o resíduo.

\section{Referências}

AMERICAN PUBLIC HEALTH ASSOCIATION, APHA. Standard methods for the examination of water and wastewater. 21st ed, Washington, DC: APHA, 2005.

AMORIM, C.C. et al. Remoção de corantes têxteis através da adsorção em resíduo do alto forno da indústria siderúrgica. In: $24^{\circ}$ CONGRESSO BRASILEIRO DE ENGENHARIA SANITÁRIA E AMBIENTAL. Belo Horizonte: Abes, II-375, 2007

ANDREOZZI, R.; D'APUZZO, A.; MAROTTA, R. Oxidation of aromatic substrates in water/goethite slurry by means of hydrogen peroxide. Water Research, v. 36, n. 19, p. 4691-4698, 2002.

AZBAR, N.; YONAR, T.; KESTIOGLU, K. Comparison of various advanced oxidation processes and chemical treatment methods for COD and color removal from polyester and acetate fiber dyeing effluent. Chemosphere, v. 55, n. 1 , p. $35-43,2004$.

CENTI, G. et al. Catalytic wet oxidation with $\mathrm{H}_{2} \mathrm{O}_{2}$ of carboxylic acids on homogeneous and heterogeneous Fenton-type catalysts. Catalysis Today, v. 55, n. 1-2, p. 61-69, 2000
CHOU, S.; HUANG, C.; HUANG, Y.H. Heterogeneous and homogeneous catalytic oxidation by supported $\gamma-\mathrm{FeOOH}$ in a fluidized-bed reactor: kinetic approach. Environmental Science \& Technology, v. 35, n. 6, p. 1247-1251, 2001.

CIÇEK, F. et al. Low cost removal of reactive dyes using wheat bran. Journal of Hazardous Materials, v. 146, n. 1-2, p. 408-416, 2007.

DABROWSKI, A. Adsorption -- from theory to practice. Advances in Colloid and Interface Science, v. 93, n. 1-3, p. 135-224, 2001.

DANTAS, T.L.P. et al. Treatment of textile wastewater by heterogeneous Fenton process using a new composite $\mathrm{Fe}_{2} \mathrm{O}_{3}$ /carbon. Chemical Engineering Journal, v. 118, n. 1-2, p. 77-82, 2006.

GALINDO, C.; JACQUES, P.; KALT, A. Photochemical and photocatalytic degradation of an indigolid dye: a case study of acid blue 74 (AB74). Journal of Photochemistry and Photobiology A: Chemistry. v. 141, p. 47-56, 2001.

HSUEH, C.L. et al. Degradation of azo dyes using low iron concentration of Fenton and Fenton-like system. Chemosphere, v. 58, n. 10, p. 1409$1414,2005$. 
JIRASRIPONGPUN, $\quad K$.; $\quad$ NASANIT, $\quad$ R.; NIRUNTASOOK, J.; CHOTIKASATIAN, B. Decolorization and degradation of Reactive Red 195. Thammasat International Journal of Science and Technology, vol. 12, n. 4, p. 6-11, 2007.

LEE, J.W. et al. Evaluation of the performance of adsorption and coagulation processes for the maximum removal of reactive dyes. Dyes and Pigments, v. 69, n. 3, p. 196-203, 2006.

LUCAS, M.S.; PERES, J.A. Decolorization of the azo dye reactive black 5 by Fenton and photo-Fenton oxidation. Dyes and Pigments, v. 71, n. 3 , p. 236-244, 2006.

MECOZZI, R. et al. Use of EAF dust as heterogeneous catalyst in Fenton oxidation of PCP contaminated wastewaters. Journal of Hazardous Materials v. 137, n. 2, p. 886-892, 2006.

MUROV, S. L. Handbook of Photochemistry. New York: Marcel Dekker, 1973.

NIGAM, P. et al. Physical removal of textile dyes from effluents and solidstate fermentation of dye-adsorbed agricultural residues. Bioresource Technology, v. 72, n. 3, p. 219-226, 2000.

OLIVER, J.H.; HYUNOOK, K.; PEN-CHI, C. Decolorization of wastewater. Critical review. Environmental Science \& Technology, v. 30, n. 4, p. 499505, 2000.

ÓRFÃO, J.J.M. et al. Adsorption of a reactive dye on chemically modified activated carbons-Influence of $\mathrm{pH}$. Journal of Colloid and Interface Science, v. 296, n. 2, p. 480-489, 2006.

PAPIC, S. et al. Removal of some reactive dyes from synthetic wastewater by combined $\mathrm{Al}(\mathrm{III})$ coagulation/carbon adsorption process. Dyes and Pigments, v. 62, n. 3, p. 291-298, 2004.
RIVERA-UTRILLA, J. et al. Activated carbon surface modifications by adsorption of bacteria and their effect on aqueous lead adsorption. Journal of Chemical Technology \& Biotechnology, v. 76, n. 12, p. 1209, 2001.

ROBINSON, T. et al. Remediation of dyes in textile effluent: a critical review on current treatment technologies with a proposed alternative. Bioresource Technology, v. 77, n. 3, p. 247-255, 2001.

SAUER, T. et al. Kinetics of photocatalytic degradation of reactive dyes in a TiO2 slurry reactor. Journal of Photochemistry and Photobiology A: Chemistry, v. 149, n. 1-3, p. 147-154, 2002.

SILVA, C.G.; WANG, W.; FARIA, J.L. Photocatalytic and photochemical degradation of mono-, di- and tri-azo dyes in aqueous solution under UV irradiation. Journal of Photochemistry and Photobiology A: Chemistry, v. 181, n. 2-3, p. 314-324, 2006.

SLEIMAN, M. et al. Photocatalytic degradation of azo dye Metanil Yellow: Optimization and kinetic modeling using a chemometric approach. Applied Catalysis B: Environmental, v. 77, n. 1-2, p. 1-11, 2007.

ŠOSTAR-TURK, S.; SIMONIČ, M.; PETRINIĆ, I. Wastewater treatment after reactive printing. Dyes and Pigments, v. 64, n. 2, p. 147-152, 2005.

TARR, M.A. Chemical degradation methods for wastes and pollutants environmental and industrial applications. New York: Marcel Dekker, 2003.

WANG, S.; ZHU, Z.H. Characterisation and environmental application of an Australian natural zeolite for basic dye removal from aqueous solution. Journal of Hazardous Materials, v. 136, n. 3, p. 946-952, 2006.

ZAGHBANI, N.; HAFIANE, A.; DHAHBI, M. Separation of methylene blue from aqueous solution by micellar enhanced ultrafiltration. Separation and Purification Technology, v. 55, n. 1, p. 117-124, 2007. 